\title{
Long-Term Assessment of the Effects of COVID-19 and Isolation Care on Survivor Disability and Anxiety
}

David M. Brett-Major, ${ }^{1{ }^{*}}$ David S. Cates, ${ }^{1}$ James V. Lawler, ${ }^{1}$ Chad Vokoun, ${ }^{1}$ Angela L. Hewlett, ${ }^{1}$ Daniel W. Johnson, ${ }^{1}$ Elizabeth R. Schnaubelt, ${ }^{1,2}$ Michael C. Wadman, ${ }^{1}$ Christopher J. Kratochvil, ${ }^{1}$ and M. Jana Broadhurst ${ }^{1}$

${ }^{1}$ University of Nebraska Medical Center and Nebraska Medicine, Omaha, Nebraska; ${ }^{2}$ United States Air Force School of Aerospace Medicine, Dayton, Ohio

Abstract. We conducted an assessment of disability, anxiety, and other life impacts of COVID-19 and isolation care in a unique cohort of individuals. These included both community admissions to a university hospital as well as some of the earliest international aeromedical evacuees. Among an initial 16 COVID-19 survivors that were interviewed 6-12 months following their admission into isolation care, perception of their isolation care experience was related to their reporting of long-term consequences. However, anxiety and disability assessed with standard scores had no relationship with each other. Both capture of the isolation care experience and caution relying on single scoring systems for assessing long-term consequences in survivors are important considerations for on-going and future COVID-19 and other pandemic survivor research.

We established a prospective, observational cohort study in February 2020, in Nebraska, at an academic referral hospital beginning with the arrival of some of the first U.S. aeromedical evacuated patients with coronavirus disease (COVID-19; UNMC IRB no. 146-20-FB). ${ }^{1}$ A full description of the cohort-to include individual level information in a line-list format-is available publically. ${ }^{2}$ To enter this cohort, participants had to present for care to our healthcare system and be suspected or confirmed to be infected by a pathogen of concern, in this case, severe acute respiratory syndrome coronavirus 2 (SARS-CoV-2). In addition to clinical evaluations in the acute and early recovery periods, participants were interviewed 6 or more months following their enrollment. These interviews were used to assess their disability, anxiety, and other life impacts from COVID-19.

Structured interviews included the World Health Organization Disability Assessment Schedule (WHODAS 2.0) 12-item scale with supplemental questions on recent days impacted; the Generalized Anxiety Disorder 7 scale (GAD-7); and openended questions on perception of life impact and reflections on their care experience transformed into binary outputs. ${ }^{3,4}$ Data handling was accomplished in REDCap ${ }^{\circledR}$ and descriptive analysis in Microsoft Excel ${ }^{\circledR}$.

Table 1 depicts line-list level demographic and survey results of the 16 COVID-19 survivors from the cohort that to date have participated in the interview. Six COVID-19 partici-

TABLE 1

Demographic factors and key survey responses of individual COVID-19 survivors*

\begin{tabular}{|c|c|c|c|c|c|c|c|c|}
\hline Age & Months since ill & $\begin{array}{l}\text { Aeromedical } \\
\text { evacuee }\end{array}$ & $\begin{array}{l}\text { Reflected } \\
\text { negatively on } \\
\text { isolation }\end{array}$ & WHODAS-12 & $\begin{array}{l}\text { Any complete } \\
\text { incapacitation or } \\
3 \text { days partial }\end{array}$ & GAD-7 & $\begin{array}{l}\text { Life changed } \\
\text { from COVID-19 }\end{array}$ & $\begin{array}{l}\text { Financial trouble } \\
\text { from COVID-19 }\end{array}$ \\
\hline $20 s$ & 12 & & & 3 & & 0 & & \\
\hline 40s & 6 & & & 0 & & 0 & & \\
\hline $40 \mathrm{~s}$ & 9 & & & 8 & & 4 & & \\
\hline 50s & 6 & & & 16 & & 4 & & \\
\hline $50 s$ & 9 & & & 18 & & 11 & & \\
\hline $60 \mathrm{~s}$ & 6 & & & 9 & & 0 & & \\
\hline $60 \mathrm{~s}$ & 9 & & & 2 & & 1 & & \\
\hline $60 \mathrm{~s}$ & 9 & & & 21 & & 1 & & \\
\hline 60st & 10 & & & 22 & & 1 & & \\
\hline $70 \mathrm{~s}$ & 7 & & & 0 & & 0 & & \\
\hline $70 \mathrm{~s}$ & 9 & & & 0 & & 8 & & \\
\hline $70 s+$ & 9 & & & 2 & & 3 & & \\
\hline $70 \mathrm{~s}$ & 10 & & & 0 & & 0 & & \\
\hline $70 \mathrm{~s}$ & 10 & & & 0 & & 0 & & \\
\hline $70 s+$ & 10 & & & 1 & & 0 & & \\
\hline $70 \mathrm{~s}$ & 10 & & & 2 & & 0 & & \\
\hline
\end{tabular}

GAD = Generalized Anxiety Disorder 7 scale; WHODAS-12 = World Health Organization Disability Assessment Schedule 12-item scale. Note: The WHODAS 2.0 12-item interview administered survey results in a score of 0-60, though a score of 17 or higher generally places the respondent in the 90 th or higher percentile of disability. The GAD-7 may range from 0 to 21 , where less than 5 is considered negative, 6-9 mild, 10-14 moderate, and 15 or higher severe. * Cells with binary outcomes are shown as either blank (negative) or shaded (affirmative).

${ }^{\dagger}$ These aeromedical evacuees required intensivist-level clinical care in the Nebraska Biocontainment Unit.

*Address correspondence to David Brett-Major, Department of Epidemiology, College of Public Health, University of Nebraska Medical Center, Nebraska Medical Center, Omaha, NE 681984395. E-mail: david.brettmajor@unmc.edu 
Box 1

Participant perceptions on isolation care and longer-term wellness*

What recommendations do you have for hospital leadership to improve the patient experience in isolation care? (WHODAS-12| GAD-7 scores)

- The hospital was perfect." $(0 \mid 0)$

- None. We feel very lucky. (2|0)

- Make sure the WiFi and TV works." (1|0)‡

- I think the doctors were wonderful." (22|1)†‡

- [Expressed wish that they had been updated on COVID news more often] $(0 \mid 0)$

- .... terrible. I could never catch my breath and couldn't do my day-to-day activities and felt like there wasn't help because people were scared to help me." (2|1)

- [Was] offered wash cloth and toothbrush after three days. [There was] no contact or help or care, felt forgotten and needed to be checked on more. Need to be more attentive. Food was always cold and I waited nearly 24 hours to eat." (8| 4)†

- No. I was content with my phone and my laptop." (0 | 8)

- I wish it was possible to have a visitor. I understood the protocol but it was very hard. I do want to say that despite this, I want you to know [that] the medical center is really, truly fantastic." (9|0)‡

- Give them the option if they are going to have a roommate or not. I would rather be by myself than have a bad roommate. Don't force people to take the vaccine. I don't want to take it and I don't want people to force me to take it. I don't trust it." (16 | 4)†‡

- No, it was difficult but there's nothing they could really do about it." $(0 \mid 0)$

WHODAS-12 = World Health Organization Disability Assessment Schedule 12-item scale.

*Eleven of the 16 respondents provided additional comments.

† Said yes to, "Have you suffered financial hardship as a consequence of this infection?"

‡ Said yes to, "Has this illness had other long-term consequences (in terms of how you feel or how you live) than what already has been discussed?"

pants underwent repatriation (aeromedical evacuation) from international travel early in the pandemic, three of whom required hospital-based care. Ten participants were enrolled from community hospitalizations. Interviewees who were admitted from the local community were younger than those who underwent aeromedical evaluation, and experienced more long-term consequences. Anxiety scores were low across respondents, with only one individual reporting mild and one reporting moderate anxiety. Disability (WHODAS 2.0) and anxiety (GAD-7) were not correlated (Table 1).

We examined a unique combination of aeromedical evacuated and community COVID-19 cases. Those evacuated were active, older adults who returned from cruise ship travel prior to known spread of the disease in the United States. Not all of these evacuees would have presented for clinical care in their own community. At the time, the federal government required them to be placed into either the National Quarantine Unit or Nebraska Biocontainment Unit, depending upon the level of care required. Both units are specially designed for quarantine and isolation, respectively. The care teams that work in these units are experienced in isolation care and managing patient needs in the setting of high consequence pathogens such as Ebola virus. In contrast, admissions from the community received care in usual healthcare spaces adapted for the emergency, with broader participation by a variety of healthcare teams new to isolation care for COVID-19 but well-practiced in the management of ill patients.

Larger cohorts should address patient perceptions and mental health in parallel with disability scoring to test the lack of association observed in our pilot group. Studies performed during or shortly following isolation care for COVID19 showed that roughly one in six persons rated their own mental health as poor or have more than moderate anxiety. ${ }^{5,6}$ Despite generally low anxiety scores in our cohort, a perception of long-term functional debility was common. Half of those reporting long-term disability (four of eight) reflected negatively on their isolation experience (Box 1). This is an intriguing early finding deserving of further investigation. Another area for further study is the financial impact among those admitted from the community.

Despite being compelled into isolation care, in our small pilot study, the perception of our aeromedical evacuation patients on their experience was no worse or better than community admissions who had presented to a university hospital seeking care. Although some of this might be explained by a sense of rescue from lengthy quarantine on a cruise ship prior to travel to Nebraska, it emphasizes the importance of capturing the nature of patients' isolation experience and their perceived impacts toward a better understanding of patientcentered quality aspects of isolation care.

Received February 17, 2021. Accepted for publication June 4, 2021. Published online July 16, 2021.

Acknowledgments: This work is possible due to commitment by study participants and care teams. UNMC and Nebraska Medicine's investigative team is large and multidisciplinary, only partially represented here. Additionally, Lauren Longacre and Louis Walker conducted the interviews and, Kathleen Angell and Julia Bai revised the open data landscape. The American Society of Tropical Medicine and Hygiene has waived the Open Access fee for this article due to the ongoing COVID-19 pandemic.

Financial support: This work was accomplished with intramural funding, to include a Rapid Response award from the UNMC College of Medicine.

Authors' addresses: David M. Brett-Major, David S. Cates, James V. Lawler, Chad Vokoun, Angela L. Hewlett, Daniel W. Johnson, Michael C. Wadman, Christopher J. Kratochvil, and M. Jana Broadhurst, University of Nebraska Medical Center and Nebraska Medicine, Omaha, NE, E-mails: david.brettmajor@unmc.edu, dcates@nebraskamed.com, james.lawler@unmc.edu, cvokoun@unmc.edu, alhewlett@unmc.edu, dan.johnson@unmc.edu, mwadman@unmc.edu, ckratoch@unmc. edu, and jana.broadhurst@unmc.edu. Elizabeth R. Schnaubelt, University of Nebraska Medical Center and Nebraska Medicine, Omaha, NE, and United States Air Force School of Aerospace Medicine, Dayton, OH, E-mail: elizabeth.schnaubelt@us.af.mil.

This is an open-access article distributed under the terms of the Creative Commons Attribution (CC-BY) License, which permits unrestricted use, distribution, and reproduction in any medium, provided the original author and source are credited.

\section{REFERENCES}

1. Brett-Major DM et al., 2020. Advanced preparation makes research in emergencies and isolation care possible: the case of novel coronavirus disease (COVID-19). Am J Trop Med Hyg 102: 926-931.

2. Broadhurst MJ, Brett-Major DM, 2020. Clinical Characterization Protocol for Severe Emerging Infections (CCPSEI). Omaha, 
NE: UNMC. Available at: https://www.unmc.edu/publichealth/ departments/epidemiology/CCPSEl.html.

3. WHO, 2012. Measuring Health and Disability: Manual for WHO Disability Assessment Schedule (WHODAS 2.0). Geneva, Switzerland: World Health Organization.

4. Spitzer RL, Kroenke K, Williams JB, Lowe B, 2006. A brief measure for assessing generalized anxiety disorder: the GAD-7. Arch Intern Med 166: 1092-1097.
5. Kang E, Lee SY, Kim MS, Jung H, Kim KH, Kim KN, Park HY, Lee YJ, Cho B, Sohn JH, 2021. The psychological burden of COVID-19 stigma: evaluation of the mental health of isolated mild condition COVID-19 patients. J Korean Med Sci 36: e33.

6. Jacobs LG et al., 2020. Persistence of symptoms and quality of life at 35 days after hospitalization for COVID-19 infection. PLOS One 15: e0243882. 